\title{
SARS-CoV-2 infects cells of the human exocrine and endocrine pancreas and interferes with beta-cell function
}

Janis A. Müller

Ulm University Medical Center

Rüdiger Groß

Ulm University Medical Center

Carina Conzelmann

Ulm University Medical Center

Jana Krüger

Ulm University Hospital

Lennart Koepke

Ulm University Medical Center

Johannes Steinhart

Ulm University Hospital

Tatjana Weil

Ulm University Medical Center

Caterina Prelli Bozzo

Ulm University Medical Center

Clarissa Read

Ulm University

Giorgio Fois

Ulm University

Tim Eiseler

Ulm University Hospital

Julia Gehrmann

RWTH Aachen University

Joanne van Vuuren

Helmholtz Zentrum München

Isabel M. Wessbecher

Heidelberg University Hospital

Manfred Frick

Ulm University

Ivan G. Costa 
RWTH Aachen University

\section{Markus Breunig}

Ulm University Hospital

\section{Michael Schuster}

CeMM Research Center for Molecular Medicine of the Austrian Academy of Sciences

\section{Stefan Liebau}

Eberhard Karls University Tübingen

\section{Thomas Seufferlein}

Ulm University Hospital

\section{Steffen Stenger}

Ulm University Medical Center

\section{Albrecht Stenzinger}

University Hospital Heidelberg

\section{Patrick E. MacDonald}

University of Alberta

\section{Frank Kirchhoff}

Ulm University Medical Center

Konstantin M. J. Sparrer

Ulm University Medical Center

\section{Paul Walther}

Ulm University

\section{Heiko Lickert}

Helmholtz Zentrum München

Thomas F.E. Barth

Ulm University Hospital

Martin Wagner ( $\nabla$ martin.wagner@uniklinik-ulm.de )

Ulm University Hospital

Jan Münch ( $\nabla$ jan.muench@uni-ulm.de)

Ulm University Medical Center

Sandra Heller ( $\square$ sandra.heller@uni-ulm.de)

Ulm University Hospital

Alexander Kleger ( $\sim$ alexander.kleger@uni-ulm.de )

Ulm University Hospital

\section{Research Article}

Keywords: SARS-CoV-2, pancreatic infection, diabetes, COVID-19

Posted Date: October 23rd, 2020 
DOI: https://doi.org/10.21203/rs.3.rs-96076/v1

License: (c) (1) This work is licensed under a Creative Commons Attribution 4.0 International License. Read Full License 


\section{Abstract}

Preexisting diabetes increases the risk of a severe course of the pandemic coronavirus disease 2019 (COVID-19). Vice versa, exacerbations of a preexisting diabetes as well as new-onset diabetes have been reported upon SARS-CoV-2 infection. Thus, there is an imperative need to clarify whether human pancreatic endocrine cells organized within an islet of Langerhans are permissive for and affected by SARS-CoV-2 infection, and to elucidate the mechanisms underlying the development of diabetes upon COVID-19. Here, we (i) defined ACE2 and TMPRSS2 expression patterns in human pancreatic endocrine and exocrine cell types, (ii) employed human pancreatic islet cultures to demonstrate susceptibility to SARS-CoV-2 infection and to viral replication in $\beta$-cells, (iii) showed that SARS-CoV-2 attenuates glucosestimulated insulin secretion, and (iv) tested remdesivir as eventually effective to prevent $\beta$-cell failure. In addition, we (v) visualized viral particles replicating in endocrine pancreatic cells and define their subcellular localization patterns via transmission electron microscopy, and finally (vi) present examples of cell type specific pancreatic infection patterns of COVID-19 deceased patients. Overall, our data demonstrate that SARS-CoV-2 can infect both the exocrine and endocrine compartments of the pancreas and can perturb $\beta$-cell integrity, which might lead to an increased risk for diabetes.

\section{Introduction}

Initially, the pandemic coronavirus disease 2019 (COVID-19), caused by the severe acute respiratory syndrome coronavirus 2 (SARS-CoV-2), was considered an exclusive lung disease eventually leading to serious respiratory symptoms ${ }^{1}$. In the meantime, accumulating experimental and clinical knowledge shows that SARS-CoV-2 also causes lesions in kidneys, heart, brain, and gastrointestinal organs ${ }^{2-7}$. SARSCoV-2 tropism towards distinct tissues is governed by cellular factors expressed on target cells such as the viral entry receptor angiotensin-converting enzyme $2(\text { ACE2 })^{8}$ or the transmembrane serine protease 2 (TMPRSS2) $^{8}$. ACE2 expression within islets of Langerhans has been reported but not yet shown to allow SARS-CoV-2 entry ${ }^{9-11}$. Diabetes mellitus presents Janus-like in COVID-19: First, preexisting diabetes per se increases the risk of a severe disease, requiring more intense interventions and increasing mortality ${ }^{12,13}$. Second, severe exacerbations of a preexisting diabetes as well as new-onset diabetes have been reported ${ }^{13-16}$ and cases of non-immune mediated diabetes in previously healthy individuals become increasingly evident ${ }^{17}$. So far, it is unclear whether SARS-CoV-2 triggers immune-mediated $\beta$-cell ablation as in type 1 diabetes (T1D) or directly perturbs $\beta$-cell function leading to non-autoimmune-mediated diabetes. Recent evidence suggests that SARS-CoV-2 can infect human endocrine cells in vitro ${ }^{18}$. However, stem-cell derived immature human $\beta$-cells were employed and neither viral replication, $\beta$-cell function, viral tropism, nor putative rescue strategies were determined ${ }^{18}$. Moreover, single cell RNAsequencing performed by three individual studies did not reveal conclusive information on ACE2 and TMPRSS2 expression patterns across distinct pancreatic cell types ${ }^{9,10,19}$. Immunostaining showed even partially opposing expression patterns for ACE2 and TMPRSS2 in the exocrine and endocrine compartment of the pancreas ${ }^{11,20-22}$. Thus, there is an imperative need to clarify whether human 
pancreatic endocrine cells organized within an islet of Langerhans are permissive for and affected by SARS-CoV-2 infection, and to elucidate the mechanisms underlying the development of diabetes upon COVID-19 ${ }^{12-16}$. Finally, promising antiviral drugs to treat SARS-CoV-2 infected patients such as remdesivir, which shorten the time to recovery in adult COVID-19 patients ${ }^{23}$, warrant testing of their capacity to resolve non-pulmonary facets of COVID-19.

Here, we (i) defined ACE2 and TMPRSS2 expression patterns in human pancreatic endocrine and exocrine cell types, (ii) employed human pancreatic islet cultures to demonstrate susceptibility to SARS-CoV-2 infection and to viral replication in $\beta$-cells, (iii) showed that SARS-CoV-2 attenuates glucose-stimulated insulin secretion, and (iv) tested remdesivir as eventually effective to prevent $\beta$-cell failure. In addition, we (v) visualized viral particles replicating in endocrine pancreatic cells and define their subcellular localization patterns via transmission electron microscopy, and finally (vi) present examples of cell type specific pancreatic infection patterns of COVID-19 deceased patients.

\section{Methods}

Specific information about material and methods is provided in the supplemental information.

\section{Results}

\section{ACE2 and TMPRSS2 expression in endocrine cells and a ductal subpopulation.}

As pancreatic ACE2 and TMPRSS2 expression is currently under debate ${ }^{11,20-22}$, we initiated our validation analysis with two reference antibodies (ab15348 and ab92323, Abcam; Supplementary Table 1), which had been extensively characterized in immunofluorescence and immunohistochemistry studies (ACE2 ${ }^{24-}$ 27; TMPRSS2 ${ }^{28-32}$ ). Immunoblotting confirmed detection of the correct molecular weight (MW) of $\sim 120$ kDa in ACE2 expressing HEK293T cells (Supplementary Figure 1A). Similarly, the TMPRSS2 antibody detected proteins of $\sim 65$ and $31 \mathrm{kDa}$, consistent with glycosylated forms of full-length TMPRSS 2 and the cleaved serine protease domain, as previously reported ${ }^{33}$ (Supplementary Figure 1B). Endogenous expression of ACE2 and TMPRSS2 was detected in human lung Calu-3 cells, as well as in fresh-frozen human pancreatic tissue comprising exocrine and endocrine cell types. Notably, ACE2 expression varied across various subjects (Supplementary Figure 1C). Immunofluorescence imaging revealed similar staining patterns for ACE2 or TMPRSS2 expression in transfected HEK293T cells and in differentiated airliquid interface (ALI) cultures of primary human airway epithelial cells (HAECs) ${ }^{34}$ (Supplementary Figure 1D,E). Upon validation of antibody specificity, we analyzed ACE2 and TMPRSS2 expression in tissue sections derived from five healthy human pancreata. Fluorescent staining of both SARS-CoV-2 entry factors was observed in the islets of Langerhans. ACE2 expression was strongest in endothelial cells and in a subpopulation of cytokeratin-19 (CK19) positive ductal and acinar cells which could be distinguished per morphology, while moderate signals were observed in endocrine cells (Figure 1A, Supplementary Figure $2 A, B, C, G)$. TMPRSS2 was detected in exocrine cells with clear ductal morphology and in the endocrine compartment (Figure 1C; Supplementary Figure 2D,E,F,H). Co-staining for endocrine cell types 
and viral entry proteins revealed an heterogenous staining pattern across the five donors with varying coefficients (Figure 1B,D). Highest coefficients were found for C-peptide (C-pep) positive $\beta$-cells co-stained for ACE2 (mean: 0.39, (0.24-0.78)) and TMPRSS2 (mean: 0.72, (0.59-0.86)) (Figure 1B,D). a- and $\delta$-cells expressing either glucagon (GCG) or somatostatin (SST), respectively, revealed a smaller ACE2 (mean: 0.15, (0.07-0.23) and mean: 0.19, (0.07-0.35)), or TMPRSS2 (mean: 0.12, (0.01-0.28) and mean: 0.18, (0.07-0.42)) double-positive fraction with less variance across the five subjects (Figure 1B,D). Thus, exocrine and endocrine cells express SARS-CoV-2 entry factors and might be susceptible to infection.

\section{SARS-CoV-2 replicates in human pancreatic islets.}

To determine susceptibility to ex vivo infection, human pancreatic islets isolated from three human donors were exposed to SARS-CoV-2, and expression of viral spike (S) and nucleocapsid (N) protein as well as endocrine cell markers were analyzed. $S$ and $N$ proteins were not detected at day 1 but became readily detectable at day 3 and 5 post infection (Figure 2A,B; Supplementary Figure 3A-F). Pancreatic islets supplemented with $5 \mu \mathrm{M}$ remdesivir did not stain positive for $\mathrm{S}$ or $\mathrm{N}$ proteins, indicating suppression of SARS-CoV-2 replication (Figure 2A,B; Supplementary Figure 3A-E). Only few cells stained positive for cleaved caspase 3 (CASP3) across all conditions, suggesting no increased apoptosis at that stage (Figure 2A,B). Albeit some cells exhibited double positivity for the pancreatic hormones Cpep/chromogranin a (CHGA) and the viral proteins N/S, an observation not the case for GCG and SST, most of the SARS-CoV-2 infected cells appeared to lack hormone expression (Figure 2A,B; Supplementary Figure 3A-F). To probe lineage identity of those cells, we stained for duodenal homeobox 1 (PDX1), a marker mostly labelling endocrine cells in the adult pancreas ${ }^{35,36}$ and found that many of the infected hormone-negative cells were still positive for PDX1, suggesting that endocrine cells lose hormones upon infection (Figure 2C). Increasing intra- and extracellular viral RNA levels in islets of donors 2 and 3 in the absence of remdesivir treatment indicated progressive viral replication (Figure 2D,E). Productive viral replication in islets of all donors was confirmed by increasing infectious viral titers in the respective supernatants (Figure 2F). Upon remdesivir treatment, almost no infectious virus was detected in supernatants of islets (Figure 2F), indicating efficient inhibition. This is in line with low viral RNA levels (Figure 2D,E) and absence of $\mathrm{N}$ or $\mathrm{S}$ protein in confocal microscopy analyses (Figure 2A,B; Supplementary Figure $3 A-E)$ in the presence of remdesivir. Thus, SARS-CoV-2 replicates in pancreatic islets and can be blocked by remdesivir.

\section{SARS-CoV-2 infected endocrine cells show subcellular damage and impaired function.}

Infection of endocrine cells by SARS-CoV-2 was further analyzed using transmission electron microscopy (TEM). Pancreatic islets from human organ donors 2 and 3 were infected with SARS-CoV-2 and analyzed after 5 days ex vivo culture (Figure 3A; Supplementary Figure 4A,B). Infection of islet cells with SARS-CoV2 resulted in dilatation and vacuolization of the endoplasmic reticulum (ER) - Golgi apparatus complex 
suggestive for ER stress and Golgi-swelling ${ }^{37-39}$. These vacuoles contained viral particles showing coronavirus morphology ${ }^{37-39}$ indicating productively infected endocrine cells. The virus containing vesicles are formed in the perinuclear region and processed to the cell surface. Furthermore, infection resulted in a marked reduction of endocrine secretory vesicles which seemed to be enlarged and maintained in the perinuclear region. In contrast, we were unable to detect intracellular virus particles or morphological alterations in remdesivir-treated islet cells (Figure 3A; Supplementary Figure 4A). Thus, SARS-CoV-2 caused a damage pattern in human endocrine cells similar to previously reported TEM phenotypes of lung and gut derived cells ${ }^{37-39}$. To analyze whether SARS-CoV-2 infection and associated cell damage of the islets affects function, we assessed the islet response towards a high glucose pulse. We found that glucose-stimulated insulin secretion (GSIS) was induced in all conditions but the magnitude of induction was reduced in infected islets (Figure 3B,C). However, GSIS in SARS-CoV-2infected human islets was only marginally restored by remdesivir treatment (Figure $3 B ; C$ ). The responsiveness to glucose, however, was lower in two islet preparations, most likely due to the limitations of prolonged ex vivo culture (Supplementary Figure 4C,D). These data suggest that glucose sensitive insulin secretion of $\beta$-cells could be impaired by SARS-CoV-2 infection.

\section{Transcriptional changes in human islets after SARS-CoV-2 infection.}

To define transcriptional changes induced by SARS-CoV-2, we performed RNA-sequencing (Smart-Seq2) of uninfected and infected (with or without remdesivir) cultured human islets from two donors. First, respective transcriptomes obtained at 5 days post infection were clustered. Most of the sample variance was determined by the two islet preparations differing also in donor sex (Supplementary Figure 5A). However, transcriptomes from SARS-CoV-2 infected cells clearly separated from uninfected counterparts, while remdesivir treatment resulted in intermediate clustering (Supplementary Figure 5A). Amongst the top up-regulated genes in SARS-CoV-2-infected islets were several interferon (IFN)-stimulated genes (ISGs) such as IFITMs ${ }^{40}$, OAS2, IFI27, and ISG15, while genes linked to beta cell physiology or diabetes ${ }^{40-}$ 47 such as SYT4, PASK, PEX6, and PLCXD3 were significantly down-regulated (Supplementary Figure 5B). Of note, ISGs were not only upregulated after SARS-CoV-2-infection compared to uninfected, but also to remdesivir treated islets (Supplementary Figure 5C). Gene ontology (GO)-term analysis confirmed an initiation of a transcriptional cellular defense reaction in response to SARS-CoV-2-infection. Terms like defense response to virus and regulation of viral genome replication were strongly upregulated after SARS-CoV-2-infection (Supplementary Figure 5D). Albeit not all of these terms were depleted after remdesivir treatment, several interferon related terms like interferon alpha/beta signaling and type I interferon signaling pathway were reverted (Supplementary Figure 5F). Partial efficacy of remdesivir treatment was further supported by the specific downregulation of COVID-19 related disease terms after remdesivir treatment (Supplementary Figure 5E,G). In contrast, no terms were significantly enriched in the uninfected or remdesivir treated infected islets compared to SARS-CoV-2 infected islets. Gene set enrichment analysis (GSEA) further confirmed the enrichment of interferon signaling in SARS-CoV-2 
infected islets against uninfected and remdesivir treated infected islets (Supplementary Figure $5 \mathrm{H}-\mathrm{K}$ ). In addition, a trend indicating loss of $\beta$-cell identity as revealed by several gene sets ${ }^{48}$ as well as defects in protein secretion in virally infected islets could be detected (Supplementary Figure $5 \mathrm{H}-\mathrm{K}$ ). Vice versa, these defects were attenuated upon remdesivir treatment pointing toward a partial transcriptional rescue in accordance with our functional ex vivo experiments (Supplementary Figure $5 \mathrm{H}-\mathrm{K}$ ). Thus, on transcriptional level infected islets show innate defense reactions and loss of $\beta$-cell identity that is reduced upon remdesivir treatment.

\section{Pronounced pancreatic infection during severe COVID-19.}

Finally, we aimed to validate the relevance of these ex vivo findings for the effects of SARS-CoV-2 infection in the pancreas of infected individuals. Therefore, we obtained pancreatic specimens after autopsy of four COVID-19 deceased patients (two with type 2 diabetes, one with otherwise not specified endocrine insufficiency, one without available past medical history), which were reviewed by a pathologist based on hematoxylin and eosin stained sections (Supplementary Table 2). Staining for SARS-CoV-2 N protein detected varying numbers of positive cells in all four patients indicating robust pancreatic infection during severe COVID-19 (Figure 4A). Specifically, viral N protein was detected in some small ducts, single or grouped acinar cells as well as in endothelial cells, in agreement with ACE2 expression patterns in intra-islet vessels (Figure 4A, close ups; compare to Supplementary Figure 2-C and ${ }^{21}$ ). Notably, some neighboring cells of infected cells frequently showed faint red staining potentially pointing toward $\mathrm{N}$ protein levels crossing the detection threshold (Figure 4A). N protein positive cells (stained in red) were not randomly scattered across the human pancreas but instead occurred in clusters of infected cells indicating localized viral spread (Figure 4A,B). To probe infection of human $\beta$-cells, we performed immunohistochemical double staining for the viral $\mathrm{N}$ protein and insulin, but only observed a few double positive cells (Figure 4A, close ups marked with \#). Nevertheless, N protein positive cell clusters were located in close vicinity to the islets of Langerhans, indicating a certain degree of association between SARS-CoV-2 infection and the endocrine compartment. This was quantified by a vicinity score based on the distance between $\mathrm{N}$ protein to insulin positive cell clusters and classified in cells with a distance $<100$ $\mu \mathrm{m}$ or $\geq 100 \mu \mathrm{m}$ against a randomly calculated reference distance. On average, $51 \%$ of SARS-CoV- 2 infected cell clusters were located close to human islets, with a significant maximum of $83 \%$ and $60 \%$ in patients no. 1 and no. 4 as well as similar trends with $40 \%$ and $31 \%$ in patients no. 2 and 3 , respectively (Figure 4B,C). Again, some insulin positive cells revealed a faint red $\mathrm{N}$ protein signal pointing towards spreading infection (Figure 4D, arrow heads). In line, morphology of some of the clearly infected cells neither resembled ductal, acinar or endocrine morphology indicating a certain degree of plasticity occurring after infection. We hypothesized that relevant viral infection in $\beta$-cells could lead to perturbed hormone secretion and loss of endocrine granules as suggested by TEM and immunostaining of infected islet explants (Figures 2,3). Indeed, high $\mathrm{N}$ protein signals appeared to go along with low insulin staining intensity (Figure 4D,E, close up) supporting our hypothesis that SARS-CoV-2 infection can trigger hormone loss. To further address this, we co-stained for the $\mathrm{N}$ protein and NKX6.1, which is exclusively expressed by $\beta$-cells within the adult pancreas ${ }^{49}$. Indeed, we detected N-/NKX6.1-double positive cells in four out of 
four patients in close proximity to islets of Langerhans and SARS-CoV-2-infected cell clusters (Figure 4F; Supplementary Figure 6). This indicates that infection in $\beta$-cells might lead to loss of hormone positivity, an observation supporting the results of our ex vivo assays (Figure 2; Supplementary Figure 3). Taken together, pancreatic SARS-CoV-2 infection appears likely to occur frequently in severe cases of infection, and in a complex probably multistep process including the exocrine and endocrine compartment.

\section{Discussion}

The disease course in COVID-19 patients can be perturbed by diabetes mellitus in two ways. On the one hand, diabetes is a risk factor for severe disease ${ }^{16,17}$, and contrariwise SARS-CoV-2 infection may directly compromise glucose metabolism ${ }^{16,17}$. Specifically, ketosis and ketoacidosis were observed during and after COVID-19, both being strong clinical indicators of an absolute lack of insulin due to $\beta$-cell loss or malfunction ${ }^{14}$. Here, we (i) dissected pancreatic expression patterns of SARS-CoV-2 viral entry proteins, (ii) demonstrated permissiveness of $\beta$-cells to SARS-CoV-2 infection and replication, which results in (iii) impaired insulin secretion. Most importantly, we demonstrated the presence of viral antigen in pancreata from diabetic COVID-19 deceased patients. Thus, our data add SARS-CoV-2 to a growing list of viruses directly perturbing $\beta$-cell integrity, leading to so-called infection-related diabetes ${ }^{50}$. This finding provides the experimental framework suggested by recent clinical studies reporting autoantibody-negative insulindependent diabetes mellitus after SARS-CoV-2 infection. Nevertheless, autoimmune manifestation can also arise years after recovery ${ }^{1,12,15}$. While Coxsackie B4 or congenital rubella virus infections per se can trigger development of type 1 diabetes (T1D) ${ }^{51-53}$, the virus-mediated $\beta$-cell insult presents heterogeneous across different viruses: For instance, enterovirus infection of $\beta$-cells can lead to (i) cell death accompanied by increased proliferation in neighboring non-infected $\beta$-cells, (ii) to impaired insulin production and secretion or (iii) $\beta$-cell dedifferentiation 54,55 .

We demonstrate that endocrine cells of human pancreatic islets express ACE2 and TMPRSS2. Consistently, there was a strong ACE2 positivity of intra-islet endothelial cells as similarly reported in other studies. In contrast, these studies did not report ACE2 expression in endocrine cells ${ }^{20,21}$. However, we observed that ACE2 expression in $\beta$-cells is variable among various human subjects suggesting that these differences are not technical problems but rather donor variations ${ }^{11,18,20,21}$. Notably, the strong inter-donor variation in the frequencies of ACE2-expressing endocrine cell types could also provide a potential explanation for varying diabetic phenotypes in COVID-19 patients. According to the current state of knowledge, ACE2 expression is the major determinant of SARS-CoV-2 entry and thus organ tropism, whereas several proteases can prime the coronavirus $S$ protein $6,8,22,56,57$, albeit pancreatic TMPRSS2 expression also matched our infection pattern of deceased COVID-19 patients. Our ex vivo data employing human islets preparations revealed productive SARS-CoV-2 infection. Thus, our results agree with those of a previous study reporting SARS-CoV-2 susceptibility of human pluripotent stem cell-derived endocrine cells ${ }^{18}$. We found that ACE2 and TMPRSS2 are hardly co-expressed in $\delta$ - and a-cells. However, at low frequency these cell types could be a target of infection too. Previous data support this hypothesis ${ }^{18}$, however, the latter might be due to a fetal maturation grade of their pluripotent stem cell- 
derived origin. Future studies to comprehensively explore the precise SARS-CoV-2 infection pattern in human islets and endocrine subpopulations are still warranted.

In contrast to other model systems e.g. gut organoids ${ }^{39,58}$, our data suggest low to moderate replication of SARS-CoV-2 in pancreatic islets. We visualized SARS-CoV-2 particles inside vacuoles in the perinuclear region ${ }^{37}$ of endocrine cells by transmission electron microscopy (TEM). The most striking observation was an enlarged and vacuolized ER-Golgi intermediate compartment, similar to observations in SARSCoV-2 infected intestinal, kidney, and airway epithelial cells ${ }^{37-39}$. The hallmarks of endocrine differentiation, namely secretory granules, are displaced and reduced. However, a more comprehensive TEM-based analysis across a complete viral replication cycle in human islets as well as more samples from infected patients is required to draw more definite conclusions. Nevertheless, the TEM observations are in line with the impaired insulin secretion observed in our study albeit we faced experimental variations across the three investigated islet preparations. Of note, e.g. $\beta$-cells infected by enterovirus display decreased glucose-stimulated insulin secretion and loss of Golgi structure ${ }^{59}$. However, also dedifferentiation of $\beta$-cells mimicking reversal to a progenitor state accompanied by decreased $\beta$-cellspecific gene transcription may occur after viral ${ }^{54}$ but also chemical ${ }^{60}$ injury. Our RNA-sequencing, confocal microscopy analysis and TEM data would be in line with both hypotheses namely ER stress followed by $\beta$-cell degranulation and dedifferentiation. However, pancreatic virus-induced injury is also a self-potentiating damage driver due to cytokine release. In fact, SARS-CoV-2 infection provoked a broad signature of cytokines and interferon (IFN)-stimulated genes (ISGs) attributed to type I and III IFN responses in human islets. We recently showed that Interferon-induced transmembrane (IFITM) proteins promote SARS-CoV-2 infection of human lung cells ${ }^{61}$. Of note, IFITM1-3 ranged top amongst upregulated transcripts in SARS-CoV-2-infected human islets. Similar gene ontology (GO)-terms have been reported in gut-derived organoids after SARS-CoV-2 infection ${ }^{39}$ identifying such intrinsically triggered immune response as a general feature across distinct organs during COVID-19.

Our results show that viral replication in ex vivo infected islets was efficiently inhibited by remdesivir. However, inhibition of viral replication was neither associated with an entire rescue in $\beta$-cell function nor full restoration of transcriptomes. This is most likely due to a delay in full $\beta$-cell recovery, which cannot be reached in the present experimental setting due to the deterioration of islets upon prolonged ex vivo culture. However, diabetic exacerbation or manifestation in COVID-19 patients might follow several mechanistical routes being eventually transient, as dedifferentiated or hormone-negative $\beta$-cells may fully recover after immune clearance of the virus ${ }^{60}$.

Our investigation of deceased COVID-19 patients and their pancreatic infection pattern sheds light on an obviously complex and multilayered infection process. The most striking observation in all four investigated samples was the scattered distribution of the infected cell clusters across the pancreas, most visible in the exocrine compartment but with a high vicinity to the islets of Langerhans in four patients. Such pattern faithfully recapitulates spread to neighboring pancreatic cells originating from a few infected cells eventually reached by viral particles directly via the blood stream during temporary 
viremia typically occurring in severe COVID-1962,63. Of note, systemic dissemination of genomic material of SARS-CoV-2 is associated with a sepsis-like biological response and critical illness in patients with COVID-1963. While insulin-positive $\beta$-cells in vivo showed only subtle signs of SARS-CoV-2 infection, the $\beta$ cell lineage label NKX6.1 clearly confirmed infection occurring in $\beta$-cells in all four investigated COVID-19 patients. Notably, pancreatic NKX6.1 expression is unique as no other transcription factor is restricted exclusively to $\beta$-cells within the adult pancreas ${ }^{49}$. As we also observed those hormone-negative cells in our human islet preparations, SARS-CoV-2 infection likely perturbs hormone-positivity by cytokine and/or ER stress followed by $\beta$-cell degranulation and dedifferentiation. Still, further analysis is necessary to fully understand the underlying pathomechanism. Robust infection of pancreatic ducts and acinar cells further explains elevated lipase levels and acute edematous pancreatitis in COVID-19 patients with SARS-CoV-2 associated pneumonia $5,15,64$.

Overall, our data demonstrate that SARS-CoV-2 can infect both the exocrine and endocrine compartments of the pancreas and can perturb $\beta$-cell integrity, which might lead to an increased risk for diabetes. Treatment with remdesivir during ex vivo infection of islets with SARS-CoV-2 inhibited viral replication and partially restored $\beta$-cell integrity.

\section{Declarations}

Conflict of interest statement: The authors have no conflicts of interest to declare.

\section{Author contributions}

J.A.M., R.G., C.C., A.K., M.W., J.M., S.H. and J.K. acquired, analyzed and interpreted data, drafted and revised the work. J.A.M, C.C., and T.W. performed and analyzed infection experiments and functional islet assays. R.G. and T.W. performed qPCR. C.P.B. performed western blots. L.K., S.H., T.E., M.W., J.K., R.G., K.M.J.S. and T.E. performed confocal imaging of stained organoids, deconvolution and editing of microscopy pictures and revised the work. C.R., J.A.M., P.W., and M.W. prepared samples for and performed electron microscopy. G.F. and M.F. performed HAEC cultures and microscopy. A.S. and I.W. provided histopathological sections from deceased COVID-19 patients. M.B., I.G.C., J.G. and M.S. performed bioinformatics analysis. J.v.V., P.E.M. and H.L. provided pancreatic islets and helped with analysis. T.F.E.B., M.W., and J.S. provided sections of human pancreatic tissue for immunofluorescence and performed double immunohistochemistry stainings and helped with analysis. S.S. supervised BSL3 work and F.K. provided resources. T.S. and M.W. helped to interpret data. S.H., M.W., A.K., and J.M. directed the work, interpreted the data and drafted the manuscript with input from all authors.

\section{Acknowledgements}

The authors would like to thank Aref Saed, Rashmi Gowdru Bijegatte, Katrin Köhn, Daniela Krnavek, Nicola Schrott, Renate Kunz, and Juliane Nell for their excellent technical assistance. We also thank Michael Melzer, Jessica Merkle, and Meike Hohwieler for helpful discussions. We thank James Lyon, Nancy Smith and Dr. Jocelyn Manning Fox (Alberta Diabetes Institute IsletCore) for their work isolating 
human islets, and organ procurement organizations across Canada, particularly the Human Organ Procurement and Exchange (HOPE) program in Edmonton and the Trillium Gift of Life Network (TGLN) in Ontario, for their work in obtaining human pancreas for research. We thank Isabel Wessbecher coordinating the DZIF tissue bank in Heidelberg for providing pancreatic tissue sections. Moreover, we thank Kei Sato and Meta Volcic for providing pLV-EF1a -human ACE2-IRES-puro and the ACE2-expressing HEK293T cells. R.G., C.C., J.K., L.K., T.W. are part of and R.G. is funded by a scholarship from the International Graduate School in Molecular Medicine Ulm. T.E. acknowledges funding by the DFG (\#380319649, \#376202546). J.A.M. is indebted to the Baden-Württemberg Stiftung for the financial support of this research project Eliteprogramme for Postdocs. This work was supported by grants from the MWK Baden-Württemberg (to J.A.M., F.K., J.M. and A.K), the BMBF (Restrict SARS-CoV-2 to F.K.) the EU's Horizon 2020 research and innovation program (Fight-nCoV, 101003555 to J.M.) and by the DFG (SPP1923 to K.S. and F.K., CRC1279 to K.S., S.S., F.K. and J.M.). I.G.C. received funding by the Excellence Initiative of the German federal and state governments.

\section{References}

1 Zhu, N. et al. A novel coronavirus from patients with pneumonia in China, 2019. New England Journal of Medicine (2020).

2 Lai, C.-C., Ko, W.-C., Lee, P.-I., Jean, S.-S. \& Hsueh, P.-R. Extra-respiratory manifestations of COVID19. International Journal of Antimicrobial Agents, 106024 (2020).

3 Gupta, A. et al. Extrapulmonary manifestations of COVID-19. Nat Med 26, 1017-1032, doi:10.1038/s41591-020-0968-3 (2020).

4 Wichmann, D. et al. Autopsy Findings and Venous Thromboembolism in Patients With COVID19: A Prospective Cohort Study. Ann Intern Med, doi:10.7326/M20-2003 (2020).

5 Xiao, F. et al. Evidence for Gastrointestinal Infection of SARS-CoV-2. Gastroenterology 158, 18311833 e1833, doi:10.1053/j.gastro.2020.02.055 (2020).

6 Puelles, V. G. et al. Multiorgan and Renal Tropism of SARS-CoV-2. N Engl J Med, doi:10.1056/NEJMc2011400 (2020).

7 Song, E. et al. Neuroinvasion of SARS-CoV-2 in human and mouse brain. bioRxiv (2020).

8 Hoffmann, M. et al. SARS-CoV-2 cell entry depends on ACE2 and TMPRSS2 and is blocked by a clinically proven protease inhibitor. Cell (2020).

9 Liu, F. et al. ACE2 Expression in Pancreas May Cause Pancreatic Damage After SARS-CoV-2 Infection. Clin Gastroenterol Hepatol, doi:10.1016/j.cgh.2020.04.040 (2020). 
10 Yang, J. K., Lin, S. S., Ji, X. J. \& Guo, L. M. Binding of SARS coronavirus to its receptor damages islets and causes acute diabetes. Acta Diabeto/ 47, 193-199, doi:10.1007/s00592-009-0109-4 (2010).

11 Fignani, D. et al. SARS-CoV-2 receptor Angiotensin I-Converting Enzyme type 2 is expressed in human pancreatic islet $\beta$-cells and is upregulated by inflammatory stress. bioRxiv (2020).

12 Zhu, L. et al. Association of blood glucose control and outcomes in patients with COVID-19 and pre-existing type 2 diabetes. Cell metabolism (2020).

13 Williamson, E. J. et al. OpenSAFELY: factors associated with COVID-19 death in 17 million patients. Nature, doi:10.1038/s41586-020-2521-4 (2020).

14 Pan, L. et al. Clinical characteristics of COVID-19 patients with digestive symptoms in Hubei, China: a descriptive, cross-sectional, multicenter study. The American journal of gastroenterology 115 (2020).

15 Wang, F. et al. Pancreatic Injury Patterns in Patients With Coronavirus Disease 19 Pneumonia. Gastroenterology, doi:10.1053/j.gastro.2020.03.055 (2020).

16 Rubino, F. et al. New-Onset Diabetes in Covid-19. N Eng/ J Med, doi:10.1056/NEJMc2018688 (2020).

17 Hollstein, T. et al. Autoantibody-negative insulin-dependent diabetes mellitus after SARS-CoV-2 infection: a case report. Nature Metabolism, doi:10.1038/s42255-020-00281-8 (2020).

18 Yang, L. et al. A Human Pluripotent Stem Cell-based Platform to Study SARS-CoV-2 Tropism and Model Virus Infection in Human Cells and Organoids. Cell Stem Cell (2020).

19 Lee, J. J. et al. Relative Abundance of SARS-CoV-2 Entry Genes in the Enterocytes of the Lower Gastrointestinal Tract. Genes (Basel) 11, doi:10.3390/genes11060645 (2020).

20 Coate, K. C. et al. SARS-CoV-2 Cell Entry Factors ACE2 and TMPRSS2 are Expressed in the Pancreas but Not in Islet Endocrine Cells. bioRxiv (2020).

21 Kusmartseva, I. et al. ACE2 and SARS-CoV-2 Expression in the Normal and COVID-19 Pancreas. bioRxiv (2020).

22 Hikmet, F. et al. The protein expression profile of ACE2 in human tissues. Mol Syst Biol 16, e9610, doi:10.15252/msb.20209610 (2020).

23 Beigel, J. H. et al. Remdesivir for the Treatment of Covid-19 - Preliminary Report. N Engl J Med, doi:10.1056/NEJMoa2007764 (2020).

24 Nicin, L. et al. Cell type-specific expression of the putative SARS-CoV-2 receptor ACE2 in human hearts. Eur Heart J 41, 1804-1806, doi:10.1093/eurheartj/ehaa311 (2020). 
25 Chen, Y. et al. Rhesus angiotensin converting enzyme 2 supports entry of severe acute respiratory syndrome coronavirus in Chinese macaques. Virology 381, 89-97, doi:10.1016/j.virol.2008.08.016 (2008).

26 Marques, F. Z. et al. Molecular characterization of renin-angiotensin system components in human intrauterine tissues and fetal membranes from vaginal delivery and cesarean section. Placenta 32, 214-221, doi:10.1016/j.placenta.2010.12.006 (2011).

27 Smits, S. L. et al. Distinct severe acute respiratory syndrome coronavirus-induced acute lung injury pathways in two different nonhuman primate species. $J$ Viro/ 85, 4234-4245, doi:10.1128/JVI.02395-10 (2011).

28 Esumi, M. et al. Transmembrane serine protease TMPRSS2 activates hepatitis C virus infection. Hepatology 61, 437-446, doi:10.1002/hep.27426 (2015).

29 Collin, J. et al. Co-expression of SARS-CoV-2 entry genes in the superficial adult human conjunctival, limbal and corneal epithelium suggests an additional route of entry via the ocular surface. Ocul Surf, doi:10.1016/j.jtos.2020.05.013 (2020).

30 Ubuka, T., Moriya, S., Soga, T. \& Parhar, I. Identification of Transmembrane Protease Serine 2 and Forkhead Box A1 As the Potential Bisphenol A Responsive Genes in the Neonatal Male Rat Brain. Front Endocrinol (Lausanne) 9, 139, doi:10.3389/fendo.2018.00139 (2018).

31 Zmora, P. et al. Non-human primate orthologues of TMPRSS2 cleave and activate the influenza virus hemagglutinin. PLoS One 12, e0176597, doi:10.1371/journal.pone.0176597 (2017).

32 Sakaguchi, W. et al. Existence of SARS-CoV-2 Entry Molecules in the Oral Cavity. Int J Mol Sci 21, doi:10.3390/ijms21176000 (2020).

33 Lucas, J. M. et al. The androgen-regulated type II serine protease TMPRSS2 is differentially expressed and mislocalized in prostate adenocarcinoma. J Patho/ 215, 118-125, doi:10.1002/path.2330 (2008).

34 Leung, J. M. et al. ACE-2 expression in the small airway epithelia of smokers and COPD patients: implications for COVID-19. Eur Respir J 55, doi:10.1183/13993003.00688-2020 (2020).

35 Holland, A. M., Hale, M. A., Kagami, H., Hammer, R. E. \& MacDonald, R. J. Experimental control of pancreatic development and maintenance. Proc Natl Acad Sci U S A 99, 12236-12241, doi:10.1073/pnas.192255099 (2002).

36 Wu, K. L. et al. Hepatocyte nuclear factor 3beta is involved in pancreatic beta-cell-specific transcription of the pdx-1 gene. Mol Cell Bio/ 17, 6002-6013, doi:10.1128/mcb.17.10.6002 (1997). 
37 Brahim Belhaouari, D. et al. The Strengths of Scanning Electron Microscopy in Deciphering SARS-CoV-2 Infectious Cycle. Frontiers in Microbiology 11, doi:10.3389/fmicb.2020.02014 (2020).

38 Zhu, N. et al. Morphogenesis and cytopathic effect of SARS-CoV-2 infection in human airway epithelial cells. Nat Commun 11, 3910, doi:10.1038/s41467-020-17796-z (2020).

39 Lamers, M. M. et al. SARS-CoV-2 productively infects human gut enterocytes. Science (2020).

40 Semplici, F. et al. Human mutation within Per-Arnt-Sim (PAS) domain-containing protein kinase (PASK) causes basal insulin hypersecretion. J Biol Chem 286, 44005-44014, doi:10.1074/jbc.M111.254995 (2011).

41 Zhang, D. D. et al. Per-Arnt-Sim Kinase (PASK): An Emerging Regulator of Mammalian Glucose and Lipid Metabolism. Nutrients 7, 7437-7450, doi:10.3390/nu7095347 (2015).

42 Aljaibeji, H. et al. Reduced Expression of PLCXD3 Associates With Disruption of Glucose Sensing and Insulin Signaling in Pancreatic beta-Cells. Front Endocrinol (Lausanne) 10, 735, doi:10.3389/fendo.2019.00735 (2019).

43 Boonen, S. E. et al. Transient neonatal diabetes, ZFP57, and hypomethylation of multiple imprinted loci: a detailed follow-up. Diabetes Care 36, 505-512, doi:10.2337/dc12-0700 (2013).

$44 \quad$ Hoytema van Konijnenburg, E. M. M. et al. Hyperinsulinism in a patient with a Zellweger Spectrum Disorder and a 16p11.2 deletion syndrome. Mol Genet Metab Rep 23, 100590, doi:10.1016/j.ymgmr.2020.100590 (2020).

45 Huang, C. et al. Synaptotagmin 4 Regulates Pancreatic beta Cell Maturation by Modulating the $\mathrm{Ca}(2+)$ Sensitivity of Insulin Secretion Vesicles. Dev Cell 45, 347-361 e345, doi:10.1016/j.devcel.2018.03.013 (2018).

46 Aljaibeji, H. et al. Reduced Expression of PLCXD3 Associates With Disruption of Glucose Sensing and Insulin Signaling in Pancreatic $\beta$-Cells. Frontiers in Endocrinology 10, doi:10.3389/fendo.2019.00735 (2019).

47 Pae, E. K. \& Kim, G. Insulin production hampered by intermittent hypoxia via impaired zinc homeostasis. PLoS One 9, e90192, doi:10.1371/journal.pone.0090192 (2014).

48 Ackermann, A. M., Wang, Z., Schug, J., Naji, A. \& Kaestner, K. H. Integration of ATAC-seq and RNAseq identifies human alpha cell and beta cell signature genes. Mol Metab 5, 233-244, doi:10.1016/j.molmet.2016.01.002 (2016).

49 Sander, M. et al. Homeobox gene Nkx6.1 lies downstream of Nkx2.2 in the major pathway of beta-cell formation in the pancreas. Development 127, 5533-5540 (2000). 
50 WHO. Classification of diabetes mellitus. World Health Organization,

doi:https://www.who.int/publications/i/item/classification-of-diabetes-mellitus (2019).

51 De Beeck, A. O. \& Eizirik, D. L. Viral infections in type 1 diabetes mellitus-why the $\beta$ cells? Nature Reviews Endocrinology 12, 263-273 (2016).

52 Fairweather, D. \& Rose, N. R. Type 1 diabetes: virus infection or autoimmune disease? Nature immunology 3, 338-340 (2002).

53 Ruiz, P. L. et al. Pandemic influenza and subsequent risk of type 1 diabetes: a nationwide cohort study. Diabetologia 61, 1996-2004 (2018).

54 Oshima, M. et al. Virus-like infection induces human beta cell dedifferentiation. JCl Insight 3, doi:10.1172/jci.insight.97732 (2018).

55 Petzold, A., Solimena, M. \& Knoch, K. P. Mechanisms of Beta Cell Dysfunction Associated With Viral Infection. Curr Diab Rep 15, 73, doi:10.1007/s11892-015-0654-x (2015).

56 Hoffmann, M., Kleine-Weber, H. \& PöhImann, S. A Multibasic Cleavage Site in the Spike Protein of SARS-CoV-2 Is Essential for Infection of Human Lung Cells. Molecular Cell 78, 779-784.e775, doi:https://doi.org/10.1016/j.molcel.2020.04.022 (2020).

57 Millet, J. K. \& Whittaker, G. R. Host cell proteases: Critical determinants of coronavirus tropism and pathogenesis. Virus Research 202, 120-134, doi:https://doi.org/10.1016/j.virusres.2014.11.021 (2015).

$58 \quad$ Krueger, J. et al. Remdesivir but not famotidine inhibits SARS-CoV-2 replication in human pluripotent stem cell-derived intestinal organoids. bioRxiv (2020).

59 Hodik, M. et al. Enterovirus infection of human islets of Langerhans affects beta-cell function resulting in disintegrated islets, decreased glucose stimulated insulin secretion and loss of Golgi structure. BMJ Open Diabetes Res Care 4, e000179, doi:10.1136/bmjdrc-2015-000179 (2016).

60 Sachs, S. et al. Targeted pharmacological therapy restores $\beta$-cell function for diabetes remission. Nature Metabolism 2, 192-209, doi:10.1038/s42255-020-0171-3 (2020).

61 Bozzo, C. P. et al. IFITM proteins promote SARS-CoV-2 infection of human lung cells. bioRxiv (2020).

62 Morris, K. V. The Improbability of the Rapid Development of a Vaccine for SARS-CoV-2. Molecular Therapy (2020).

63 Bermejo-Martin, J. F. et al. SARS-CoV-2 RNA viremia is associated with a sepsis-like host response and critical illness in COVID-19. medRxiv (2020). 
64 Schepis, T. et al. SARS-CoV2 RNA detection in a pancreatic pseudocyst sample. Pancreatology 20, 1011-1012, doi:10.1016/j.pan.2020.05.016 (2020).

\section{Figures}

Figure 1

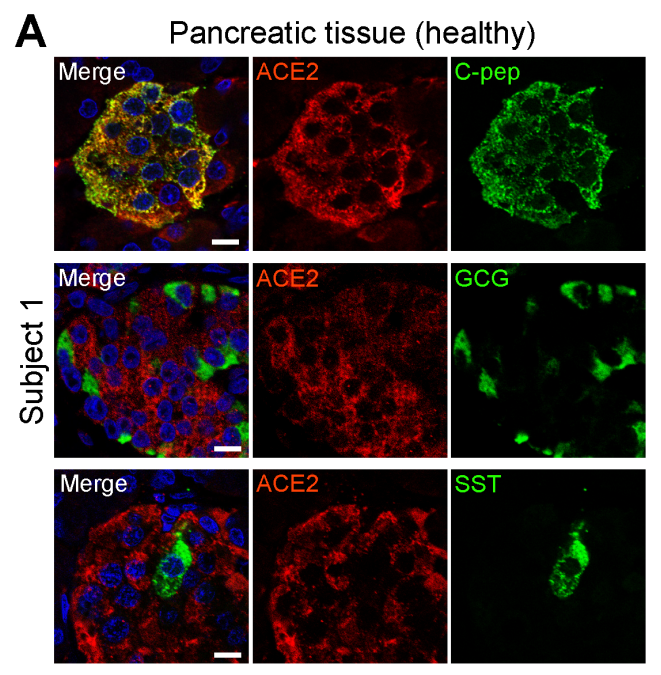

\section{B}
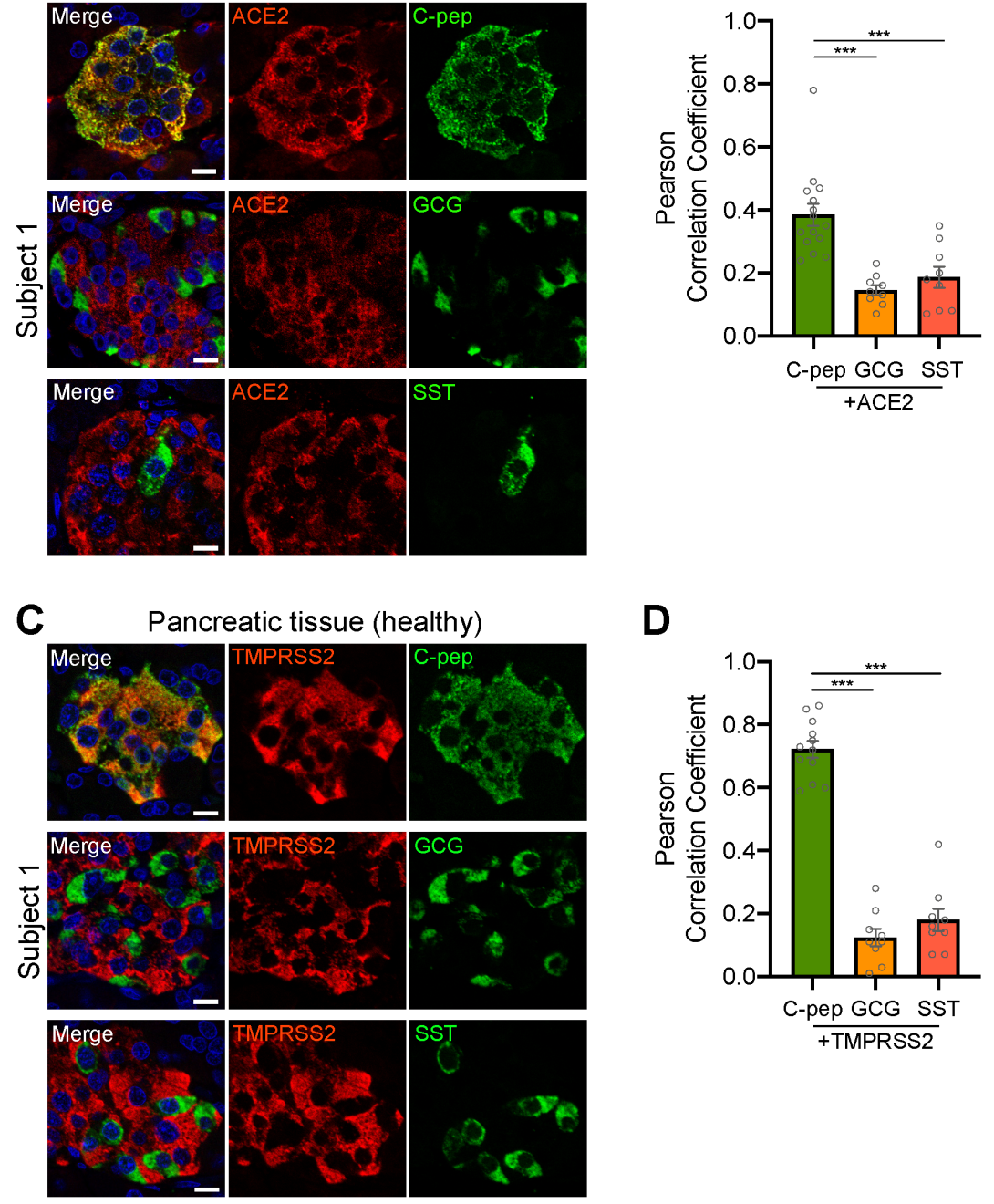

D

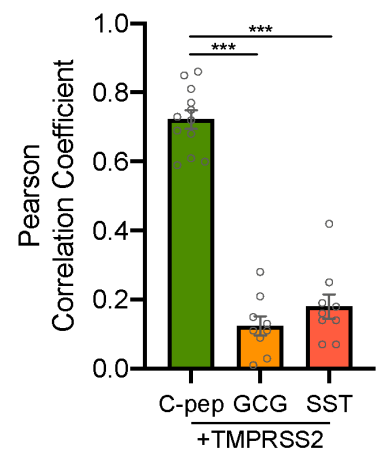

Figure 1 
Pancreatic $\beta$-cells express SARS-CoV-2 entry factors ACE2 and TMPRSS2. (A,C) Adult pancreatic tissue sections from five healthy subjects were stained with antibodies against ACE2 (A; red) or TMPRSS2 (C; red), and C-peptide (C-pep, green), glucagon (GCG, green), or somatostatin (SST, green). Cell nuclei were visualized by DAPI (blue). Representative confocal sections of subject 1 are shown, scale bars depict 10 $\mu \mathrm{m}$. (B,D) Co-localization of cell-specific markers C-pep, GCG, or SST with ACE2 (B) or TMPRSS2 (D) was analyzed by Pearson correlation coefficients using Fiji. Numbers of investigated healthy human pancreata were as follows: ACE/C-pep: $n=5$; TMPRSS2/C-pep: $n=4$; ACE2/SST or GCG: $n=3$; TMPRSS2/ SST or GCG: $n=3$. Error bars indicate standard error of coefficient quantified for each subject and staining combination. ${ }^{* \star} \mathrm{p}<0.001$, Ordinary one-way ANOVA with Tukey's post-test. 


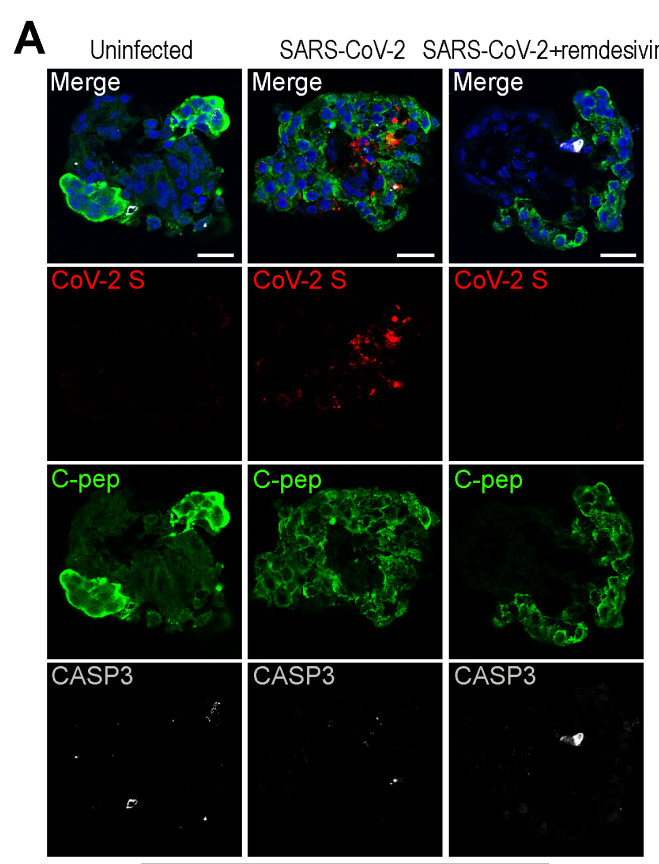

day 3

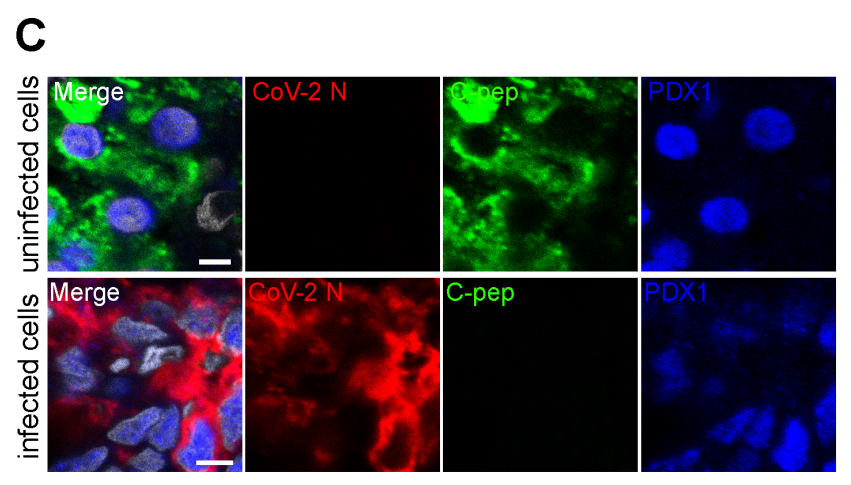

E

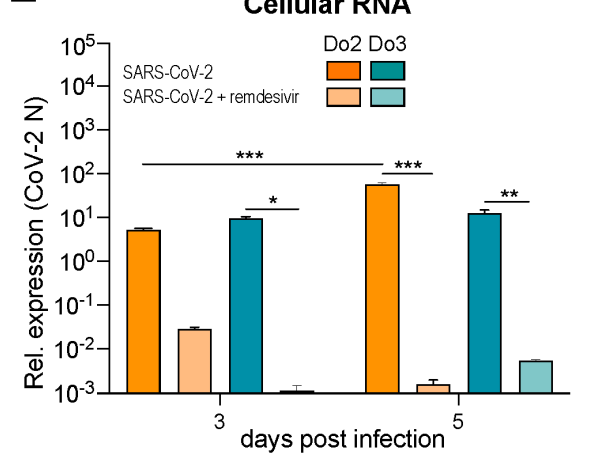

Figure 2

B

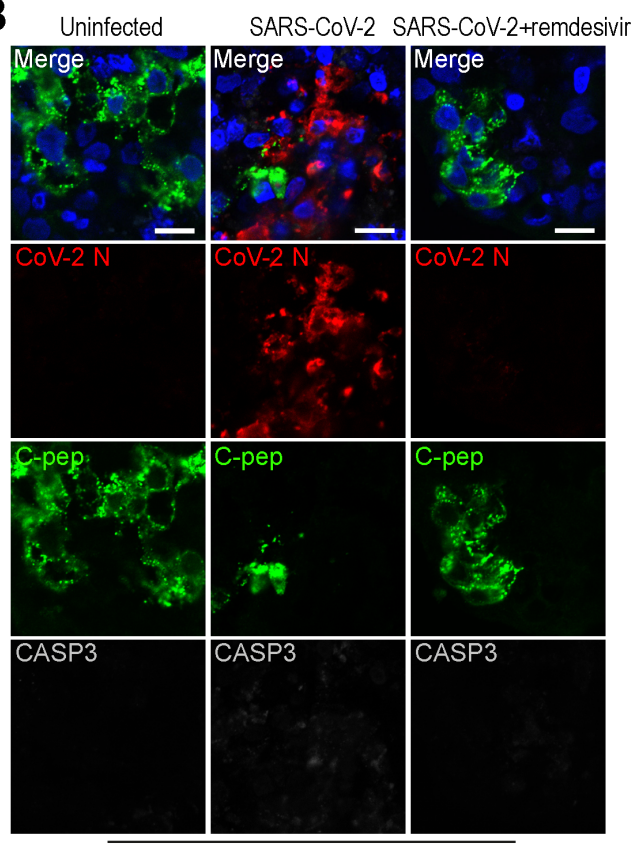

day 5

D

Viral RNA

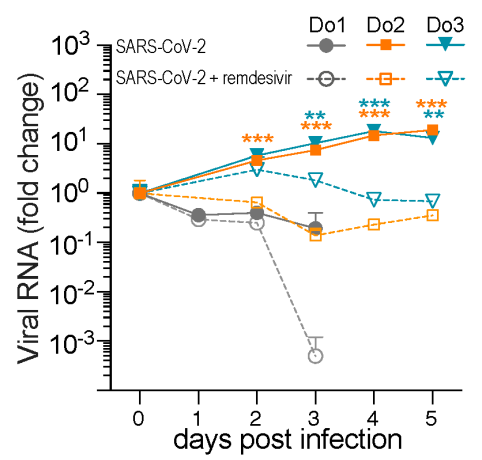

$\mathbf{F}$
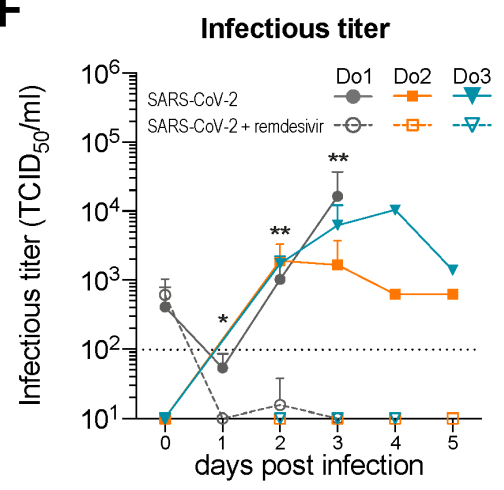

\section{Figure 2}

SARS-CoV-2 productively infects human pancreatic islets. (A-F) Human pancreatic islets were mocktreated with medium or infected with SARS-CoV-2 and cultivated in the presence or absence remdesivir (5 $\mu \mathrm{M})$. (A) Islets of donor 1 fixed after 3 days of infection were stained for SARS-CoV-2 Spike (CoV-2 S, red), C-peptide (C-pep, green), cleaved caspase 3 (CASP3, white), and nuclei (DAPI, blue). Representative images are shown; scale bars depict $20 \mu \mathrm{m}$. (B) Islets 5 days post infection (donor 2) were stained for 
SARS-CoV-2 nucleocapsid ( $\mathrm{N}$, red), C-peptide (C-pep, green) and cleaved caspase 3 (CASP3, white). Representative images are shown, scale bars: $10 \mu \mathrm{m}$. (C) Islets from donor 3 were infected and SARSCoV-2 infected cells (CoV-2 N, red), $\beta$-cells (C-pep, green) and endocrine lineage cells (PDX1, blue) visualized. Nuclei stained with DAPI are pseudocolored in white; scale bars: $5 \mu \mathrm{m}$. SARS-CoV-2-infected cells frequently became hormone-negative (C-pep) but remained lineage positive (PDX1). Uninfected cells remained double-positive for C-pep and PDX1. (D) Supernatants of islets (donor 1-3) were harvested over 5 days and SARS-CoV-2 ORF1b-nsp14 was quantified by qPCR. Error bars indicate standard deviation of 2 replicates. For each donor, changes relative to day 0 (viral input RNA) are visualized. Ordinary one-way ANOVA with Tukey's post-test of values in the presence and the absence of remdesivir at the indicated day. (E) SARS-CoV-2 N was quantified in cellular RNA isolates of donor 2 and 3 at days 3 and 5 post infection and normalized to GAPDH RNA. Error bars indicate standard deviation of 2 replicates. Ordinary one-way ANOVA with Tukey's post-test. (F) Supernatants of all 3 donors from (D) were assessed for infectivity by tissue culture infectious dose 50 (TCID50) endpoint titration. Error bars indicate standard deviation of 2 replicates and dotted line the LLOQ (lower limit of quantification). Mann Whitney test of combined donor values with and without remdesivir at the indicated day. $* \star x p<0.001, * \star p<0.01, * p<$ 0.05 . 


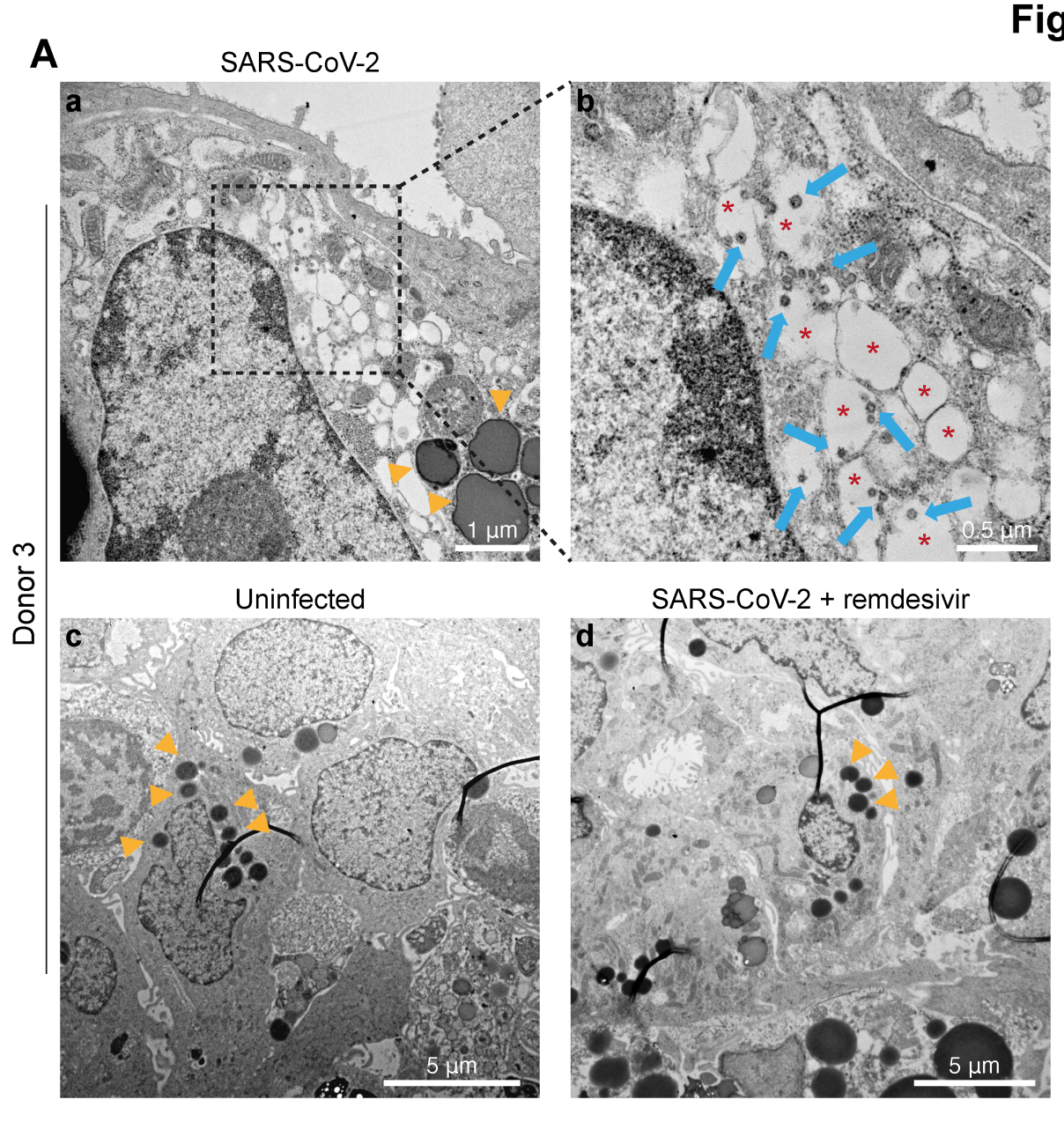

Figure 3
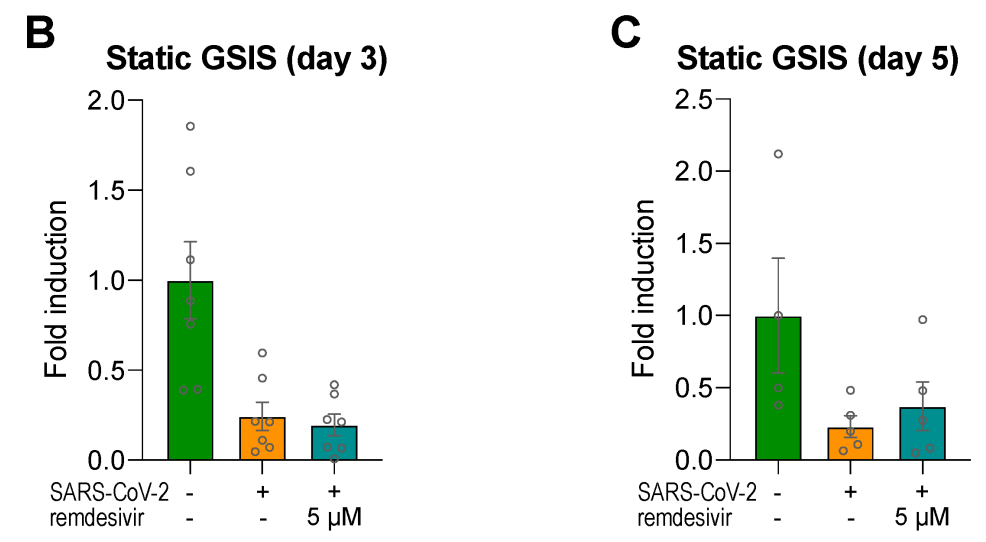

\section{Figure 3}

SARS-CoV-2 infects and replicates in pancreatic islets resulting in impairment of $\beta$-cell function. Human pancreatic islets of donor 3 were infected with SARS-CoV-2 and cultivated with or without $5 \mu \mathrm{M}$ of remdesivir, or left uninfected. (A) At day 5, islets were fixed and sectioned for transmission electron microscopy analysis. Electron micrograph (a) and magnified inlet (b) of the infected preparation show cells with endocrine secretory vesicles (yellow arrowheads) and dilated Golgi vacuoles (red asterisks) 
containing virus particles (blue arrows). Vacuoles and viral particles were absent in the uninfected (c) and remdesivir-treated $(5 \mu \mathrm{M})$ samples $(\mathrm{d})$. For micrographs illustrating normal organelles in uninfected and remdesivir-treated, SARS-CoV-2 infected cells at equal magnifications as (a-b) see Supplementary Figure 4A. (B) At day 3 (donors 1-3) and (C) day 5 (donors 2-3) post infection, islet functionality was analyzed by static glucose-stimulated insulin secretion (GSIS). Islets were exposed to first $2 \mathrm{mM}$ then $20 \mathrm{mM}$ glucose for 1 hour each, and insulin secretion into the buffer was determined by ultrasensitive insulin ELISA and normalized to values obtained at $2 \mathrm{mM}$ glucose. Error bars indicate standard error of 2-3 replicates.

Figure 4

A COVID-19 patient No. 1

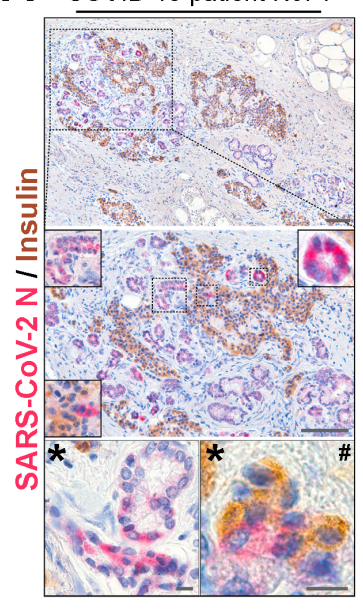

COVID-19 patient No. 2

COVID-19 patient No. 3

COVID-19 patient No. 4
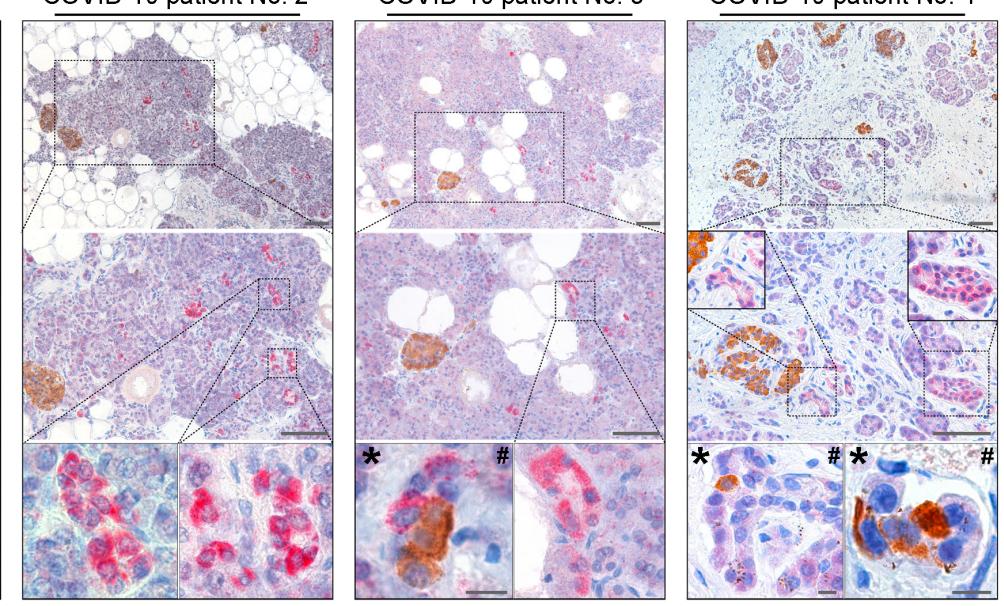

B

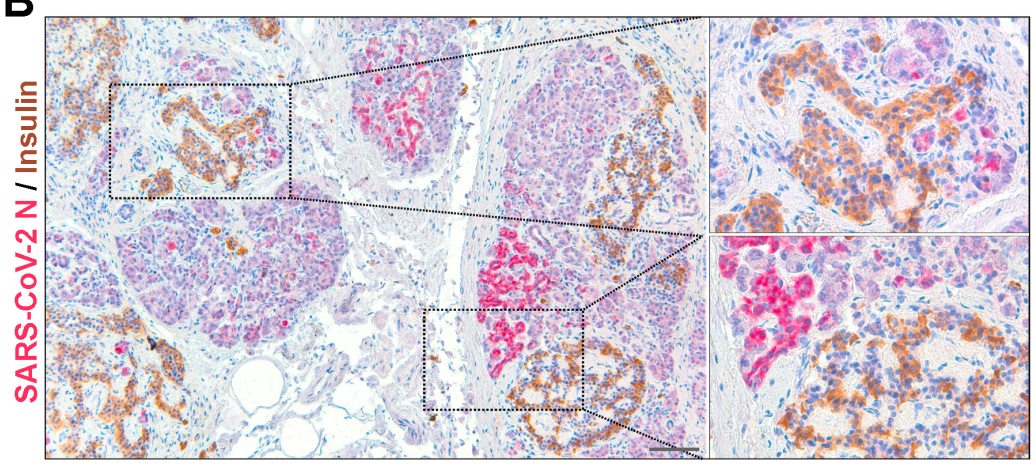

C
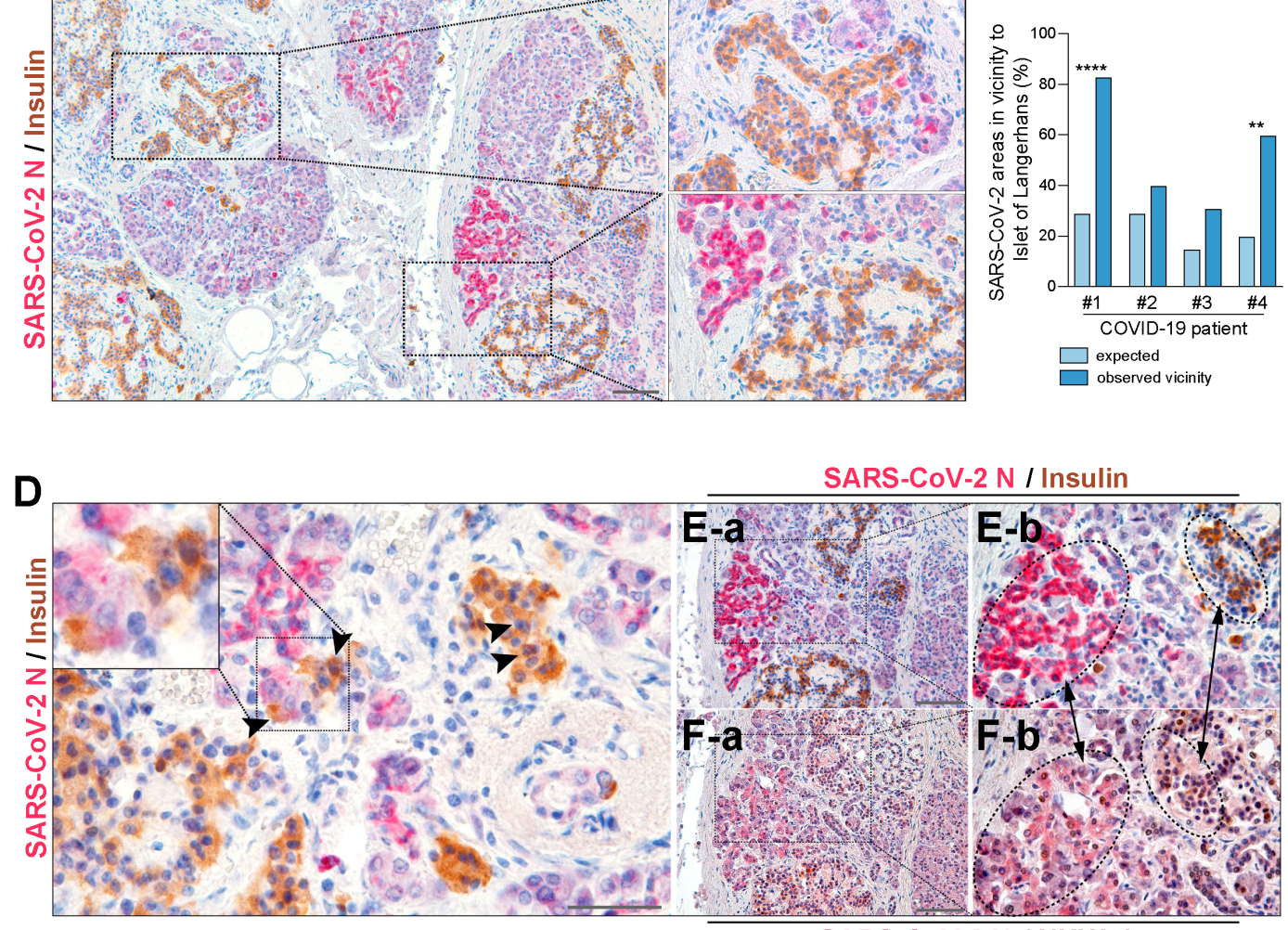

SARS-CoV-2 N / NKX6.1 


\section{Figure 4}

Pancreatic infection pattern in COVID-19 deceased patients. (A) Pancreatic tissue sections from four different COVID-19 deceased patients were stained for SARS-CoV-2 nucleocapsid ( $\mathrm{N}$; red) and insulin (brown). Rectangles mark areas of higher magnification in the next row. Inserts show further high magnification to illustrate specific patterns. Asterisks $\left(^{*}\right)$ mark magnification of stained areas outside the illustrated regions but corresponding to the patients from the respective column. Infection occurred as $\mathrm{N}$ positive clusters in all 4 patients with positivity of some ductal cells and a few acinar cells. Insulin and $\mathrm{N}$ protein double-positive cells were observed in three out of four patients (marked with \#). (B) N-positive cells are often located in close vicinity to islets of Langerhans or even mixed in islet-like structures together with insulin-positive cells. Morphology of N-positive cells frequently resembled non-acinar/nonductal morphology. Images are derived from patient No. 1. (C) Vicinity ratio of N-positive regions ( $>5$ positive cells) located nearby insulin-positive endocrine cells ( $<100 \mu \mathrm{m}$ distance to endocrine cell) divided by all $\mathrm{N}$-positive regions ( $>5$ positive cells), reveals that SARS-CoV- 2 is not randomly distributed across the pancreas, but rather located close to endocrine structures. On average, $51 \%$ of $\mathrm{N}$-positive regions are located close to endocrine cells or islets. (D) Viral N-and insulin-double-positive cells are rare (arrow heads) but interlaced into islets of Langerhans. Highest $\mathrm{N}$ protein signal in a cell cluster or even in individual cells correlates with lowest insulin signal (close up). (E,F) Consecutive sections were either stained for insulin (E) or NKX6.1 (F) together with N protein. Rectangles in (a) mark areas of higher magnification (b). Dashed areas connected with double-headed arrows mark corresponding regions which are highly N-positive, insulin-negative, and NKX6.1-positive (left) or N-negative and insulin-positive but NKX6.1-negative (right). Scale bars: $100 \mu \mathrm{m}$; inserts $10 \mu \mathrm{m}$.

\section{Supplementary Files}

This is a list of supplementary files associated with this preprint. Click to download.

- Mulleretal.SI14.10FINAL.pdf

- FiguresSupplement.pdf 